\title{
Combined subsegmentectomy: postoperative pulmonary function compared to multiple segmental resection
}

\author{
Kentaro Yoshimoto ${ }^{1}$, Hiroaki Nomori ${ }^{1}{ }^{2 *}$, Takeshi Mori ${ }^{1}$, Yasuomi Ohba ${ }^{1}$, Kenji Shiraishi $^{1}$, Koei Ikeda ${ }^{1}$
}

\begin{abstract}
Background: For small peripheral c-T1NOMO non-small cell lung cancers involving multiple segments, we have conducted a resection of subsegments belonging to different segments, i.e. combined subsegmentectomy (CSS), to avoid resection of multiple segments or lobectomy. Tumor size, location of tumor, and forced expiratory volume in 1 second $\left(\mathrm{FEV}_{1}\right)$ of each preserved lobe were compared among the CSS, resection of single segment, and that of multiple segments.

Methods: $\mathrm{FEV}_{1}$ of each preserved lobe were examined in 17 patients who underwent CSS, 56 who underwent resection of single segment, and 41 who underwent resection of multiple segments, by measuring pulmonary function and lung-perfusion single-photon-emission computed tomography and computed tomography before and after surgery.
\end{abstract}

Results: Tumor size in the CSS was significantly smaller than that in the resection of multiple segments $(1.4 \pm 0.5$ vs. $2.0 \pm 0.8 \mathrm{~cm}, \mathrm{p}=0.002$ ). Tumors in the CSS were located in the right upper lobe more frequently than those in the resection of multiple segments ( $53 \%$ vs. $5 \%, p<0.001$ ). Postoperative of FEV ${ }_{1}$ of each lobe after the CSS was higher than that after the resection of multiple segments ( $0.3 \pm 0.2$ vs. $0.2 \pm 0.2 \mathrm{l}, \mathrm{p}=0.07$ ). Mean $\mathrm{FEV}_{1}$ of each preserved lobe per subsegment after CSS was significantly higher than that after resection of multiple segments $(0.05 \pm 0.03$ vs. $0.03 \pm 0.02 \mathrm{l}, p=0.02)$. There was no significant difference of these factors between the CSS and resection of single segment.

Conclusions: The CSS is effective for preserving pulmonary function of each lobe, especially for small sized lung cancer involving multiple segments in the right upper lobe, which has fewer segments than other lobes.

\section{Background}

Advances in high-resolution CT scanning have led to frequent detection of peripheral T1N0M0 non-small cell lung cancers (NSCLCs). Several studies have demonstrated the effectiveness of segmentectomy, regarding not only preservation of pulmonary function but also prognosis [1-4]. However, for small peripheral c-T1NOM0 NSCLCs involving multiple segments, resection of entire segments damages pulmonary function to the same extent as lobectomy. To evaluate local pulmonary function, a lung-perfusion single-photonemission computed tomography (SPECT) and computed

\footnotetext{
* Correspondence: hnomori@sc.itc.keio.ac.jp

'Departments of Thoracic Surgery, Faculty of Life Sciences, Kumamoto

University, 1-1-1 Honjo, Kumamoto 860-8556, Japan

Full list of author information is available at the end of the article
}

tomography $(\mathrm{SPECT} / \mathrm{CT})$ is a reliable tool $[5,6]$. We recently examined the forced expiratory volume in 1 second $\left(\mathrm{FEV}_{1}\right)$ of each lobe after segmentectomy by using a lung-perfusion SPECT/CT. The results showed that the $\mathrm{FEV}_{1}$ of the preserved lobes after resection of 1 , 2 , and 3 segments were decreased, respectively, to $50 \%$, $35 \%$, and $17 \%$ of the preoperative value [7]. Especially, the resection of 2 segments in the right upper lobe, which has only 3 segments, can only preserve one segment. Therefore, for patients with small peripheral c-T1N0M0 NSCLCs involving multiple segments, we attempted the resection of only subsegments involved by tumor, i.e. combined subsegmentectomy (CSS), to preserve pulmonary function by avoiding the resection of multiple segments. For example, if the tumor involved the subsegment $2 \mathrm{~b}$ and $3 \mathrm{a}$ of the right upper 
lobe (Figure 1), we performed the resection of S2b and S3a subsegments. This study examined the results of CSS in patients with peripheral c-T1N0M0 NSCLCs, with special reference to tumor size, location of tumor, and postoperative pulmonary function, which were compared with that after the resection of multiple segments.

\section{Methods \\ Eligibility}

The Ethics Committees of Kumamoto University Hospital approved the study protocol for sublobar resection in patients with c-T1NOM0 NSCLC. Informed consent was obtained from all patients after a comprehensive discussion of the risks and benefits of the proposed procedures $[8,9]$.

\section{Indications for Segmentectomy and Subsegmentectomy}

The criteria for segmentectomy was the followings: (1) peripheral c-T1N0M0 NSCLCs less than $3 \mathrm{~cm}$ diameter; (2) intraoperative frozen section of lymph nodes showed no metastasis; and (3) surgical margin of at least $2 \mathrm{~cm}$ from the tumor can be taken using CSS. The CSS or multiple segmentectomy was further indicated for tumors involving multiple segments, which were identified on serial sections of the axial, sagittal, and coronal views of multidetector $\mathrm{CT}$ images using Digital Imaging and Communications in Medicine data.

\section{Combined Segmentectomy Procedure}

Segmentectomy including CSS was performed via open thoracotomy under one-lung ventilation as follows:

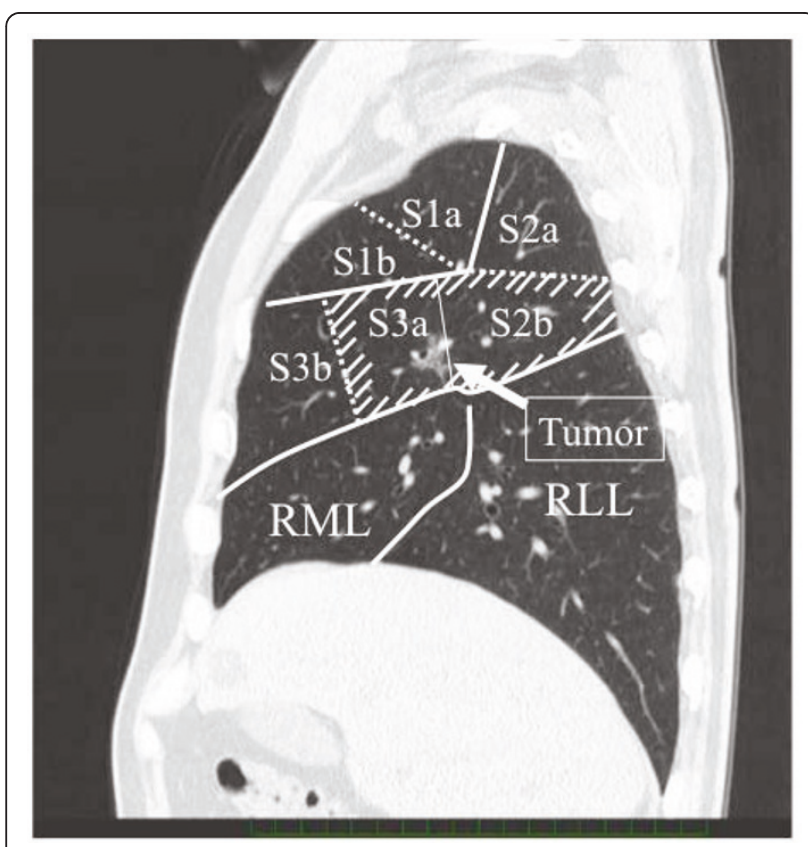

Figure 1 Sagittal image of CT. The tumor located between the right subsegment $2 \mathrm{~b}$ and $3 \mathrm{a}$.
(1) Pulmonary arteries and bronchi with tumor involvement were identified; (2) After the entire lung had been inflated, bronchi of the involved segment or subsegment were ligated and cut to clarify the boundary between the subsegments to be preserved versus resected, according to a previously reported technique [10]; (3) One lung ventilation was restarted, which made the lung tissues designated for preservation lose gas and collapse while retaining the segments subsegments designated for resection to be inflated, thereby allowing the border between the segments or subsegments of the resecting versus preserving lung tissue to be clarified; (4) The lung was cut using electrocautery between the inflated lung tissue to be resected and the deflated one to be preserved, thereby enabling the resection of targeted segments or subsegments; and (5) Cut plane of the lung was covered with a polyglycol acid sheet (Neoveil: Gunze Ltd., Kyoto, Japan) and fibrin glue to prevent postoperative air leakage.

\section{Patients}

During April 2005 - March 2009, 248 patients with c-T1N0M0 NSCLC were treated with surgery. Of them, 198 patients (79\%) were treated by segmentectomy. Of the 198 patients, CSS was conducted in 32 patients (16\%). The other 166 patients were treated by the resection of single segment (single segmentectomy, $\mathrm{n}=97$ ) or the resection of multiple segments (multiple segmentectomy, $n=69$ ).

\section{Pulmonary Function Tests}

Vital capacity (VC), forced vital capacity (FVC), and $\mathrm{FEV}_{1}$ were measured before and more than 6 months after surgery with the patient in a seated position using a dry rolling-seal spirometer (CHESTAC-9800DN; Chest Inc. Tokyo, Japan) according to American Thoracic Society standards [11].

\section{SPECT/CT}

SPECT/CT system was composed of a commercially available gantry-free SPECT with dual-head detectors (Skylight; ADAC Laboratories, Milpitas, Calif) and an 8-multidetector-row CT scanner (Light-Speed Ultra Instrument; General Electric, Milwaukee, Wis). Each $185 \mathrm{MBq}$ of $99 \mathrm{mTc}$-macroaggregated human serum albumin (Daiichi Radioisotope Laboratories, Ltd, Tokyo, Japan) was administered intravenously. The two scans were performed sequentially. The SPECT images were manually fused with the CT images on the workstation (AZE Virtual Place; AZE Co Ltd, Tokyo, Japan) $[5,7]$.

Postoperative SPECT/CT was conducted with the pulmonary function test more than 6 months after surgery.

\section{Measurement of Pulmonary Function of Each Lobe}

Images of the lobe before segmentectomy and of the lobe remaining after segmentectomy were traced on the 
CT image with a region of interest, of which radioisotope (RI) was counted on the SPECT image (Figure 2).

The $\mathrm{FEV}_{1}$ of the lobe before (A) and after (B) segmentectomy was measured from the preoperative or postoperative SPECT/CT according to the following formulae. $\mathrm{A}=$ Preoperative $\mathrm{FEV}_{1} \times[\mathrm{RI}$ counts of the lobe/RI counts of the whole lung]

$\mathrm{B}=$ Postoperative $\mathrm{FEV}_{1} \times[\mathrm{RI}$ counts of the lobe/RI counts of the whole lung]

The postoperative $\mathrm{FEV}_{1}$ of the lobe per preserved subsegment $(C)$ was measured according to the following formula.

$\mathrm{C}=\mathrm{B} /$ number of preserved subsegments of the lobe

\section{Statistical Analysis}

Student's $t$-test was used to compare the tumor size, number of the resected or preserved subsegments, and preserved $\mathrm{FEV}_{1}$ among the CSS, single segmentectomy, and multiple segmentectomy. Pearson's $\chi^{2}$ test was used to compare the location of tumors among the three groups. The SPSS software (SPSS Inc., Chicago, Illinois) was used for these analyses. Values of $p<0.05$ were accepted as significant. All values in the text and table are given as mean $\pm \mathrm{SD}$.

\section{Results}

Of the 32 patients who underwent the CSS, 17 patients who underwent both the pulmonary function test and lung-perfusion SPECT/CT both before and after surgery. Table 1 presents their resected sites and the number of

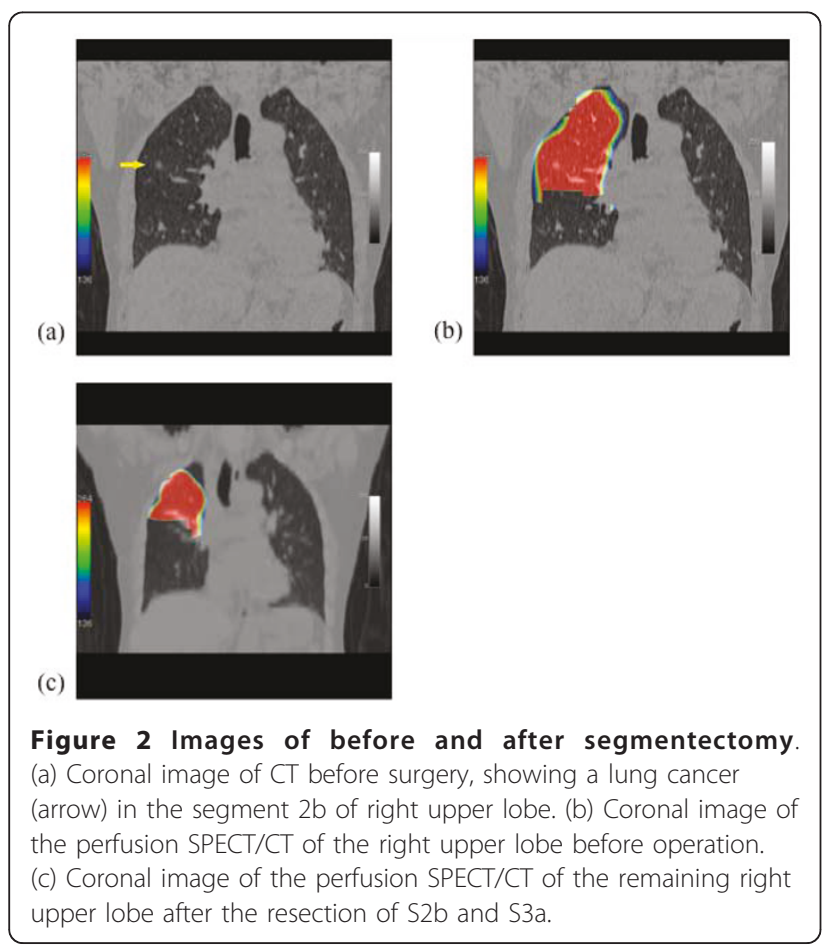

Table 1 Sites of combined subsegmentectomy and the number of resected subsegments

\begin{tabular}{|c|c|c|c|}
\hline & Resected sites & No. of resected SS & No. of patients \\
\hline \multicolumn{4}{|l|}{ Right } \\
\hline \multirow[t]{6}{*}{ Upper lobe } & $\mathrm{S} 2 \mathrm{~b}+\mathrm{S} 3 \mathrm{a}$ & 2 & 3 \\
\hline & $\mathrm{S} 2+\mathrm{S} 1 \mathrm{a}$ & 3 & 2 \\
\hline & $\mathrm{S} 1 \mathrm{a}+\mathrm{S} 2 \mathrm{~b}+\mathrm{S} 3 \mathrm{a}$ & 3 & 1 \\
\hline & $51 b+53 a$ & 2 & 1 \\
\hline & $S 1 b+53 b$ & 2 & 1 \\
\hline & $\mathrm{S} 3+\mathrm{S} 2 \mathrm{a}$ & 3 & 1 \\
\hline \multirow[t]{3}{*}{ Lower lobe } & $S 6 b+58 a$ & 2 & 1 \\
\hline & $56 b+58 a+59 a$ & 3 & 1 \\
\hline & $58 a+59 a$ & 2 & 1 \\
\hline \multicolumn{4}{|l|}{$\underline{\text { Left }}$} \\
\hline \multirow[t]{4}{*}{ Upper lobe } & $\mathrm{S} 1+2+\mathrm{S} 3 \mathrm{a}$ & 4 & 1 \\
\hline & $\mathrm{S} 1+2 \mathrm{c}+\mathrm{S} 3 \mathrm{a}$ & 2 & 1 \\
\hline & $S 1+2 c+S 3 b$ & 2 & 1 \\
\hline & $\mathrm{S} 3+\mathrm{S} 1+2 \mathrm{a}$ & 4 & 1 \\
\hline Lower lobe & $S 9+S 10+58 a$ & 6 & 1 \\
\hline Total & & & 17 \\
\hline
\end{tabular}

SS: subsegment, CSS: combined subsegmentectomy.

resected subsegments. Mean number of the resected subsegments was $2.9 \pm 1.1$. If the entire segments involved by tumors were resected, the mean number of resected subsegments would be $5.0 \pm 1.2$, i.e. the CSS could save $2.2 \pm 1.2$ subsegments compared with the resection of entire segments.

Of the 97 patients who underwent the single segmentectomy, 56 patients who underwent both the pulmonary function test and a lung-perfusion SPECT/CT both before and after surgery (Table 2). Of the 69 patients who underwent the multiple segmentectomy, 41 patients who underwent both the pulmonary function test and a lung-perfusion SPECT/CT both before and after surgery (Table 3).

Table 4 presents a comparison of preoperative pulmonary function, tumor size, location of tumor, and the numbers of resected and preserved subsegments among the CSS, single segmentectomy, and multiple segmentectomy. No significant difference of preoperative pulmonary function tests was found among these groups. Mean tumor size was $1.4 \pm 0.5 \mathrm{~cm}$ in the CSS group, which was significantly smaller than the $2.0 \pm 0.8 \mathrm{~cm}$ in multiple segmentectomy $(\mathrm{p}=0.002)$. Location of tumor in the right upper lobe was 9 of the 17 (53\%) patients who underwent the CSS, which was more frequent than 2 of the 41 (5\%) who underwent the multiple segmentectomy ( $<<0.001)$. Mean number of the resected subsegments in the CSS group was $2.9 \pm 1.1$, which was significantly less than $5.3 \pm 1.4$ of the multiple segmentectomy group $(p<0.001)$. However, the mean numbers 
Table 2 Sites of single segmentectomy and the number of resected subsegments

\begin{tabular}{|c|c|c|c|}
\hline & Resected sites & No. of resected SS & No. of patients \\
\hline \multicolumn{4}{|l|}{$\underline{\text { Right }}$} \\
\hline \multirow[t]{3}{*}{ Upper lobe } & S1 & 2 & 2 \\
\hline & $\mathrm{S} 2$ & 2 & 4 \\
\hline & S3 & 2 & 9 \\
\hline \multirow[t]{4}{*}{ Lower lobe } & S6 & 3 & 10 \\
\hline & S8 & 2 & 4 \\
\hline & S9 & 2 & 2 \\
\hline & $S 10$ & 3 & 1 \\
\hline \multicolumn{4}{|l|}{ Left } \\
\hline \multirow[t]{3}{*}{ Upper lobe } & $\mathrm{s} 1+2$ & 3 & 10 \\
\hline & S3 & 3 & 6 \\
\hline & S4 & 2 & 1 \\
\hline \multirow[t]{2}{*}{ Lower lobe } & S6 & 3 & 6 \\
\hline & S8 & 2 & 1 \\
\hline Total & & & 56 \\
\hline
\end{tabular}

of preserved subsegments of each lobe were not significantly different between the CSS and multiple segmentectomy $(5.4 \pm 2.5$ vs. $5.0 \pm 1.5)$. This discrepancy of the numbers of resected and preserved subsegments was caused by the difference of location of tumor, i.e. (1) Although the right upper lobe has only 6 subsegments, the right lower lobe, the left upper lobe, and the left lower lobe have 12, 10, and 10 subsegments, respectively, which makes the segmentectomy for the right

Table 3 Sites of multiple segmentectomy and the number of resected subsegments

\begin{tabular}{|c|c|c|c|}
\hline & Resected sites & No. of resected SS & No. of patients \\
\hline \multicolumn{4}{|l|}{ Right } \\
\hline \multirow[t]{2}{*}{ Upper lobe } & $\mathrm{S} 1+\mathrm{S} 2$ & 4 & 3 \\
\hline & $\mathrm{S} 2+\mathrm{S} 3$ & 4 & 1 \\
\hline \multirow[t]{7}{*}{ Lower lobe } & $\mathrm{S} 6+\mathrm{S} 8$ & 5 & 1 \\
\hline & $56+\$ 9$ & 5 & 1 \\
\hline & $\mathrm{S} 7+\mathrm{S} 8$ & 4 & 1 \\
\hline & $S 9+\$ 10$ & 5 & 1 \\
\hline & $\mathrm{S} 6+\mathrm{S} 9+\mathrm{S} 10$ & 8 & 1 \\
\hline & $S 8+S 9+S 10$ & 7 & 1 \\
\hline & $\mathrm{S} 7+\mathrm{S} 8+\mathrm{S} 9+\mathrm{S} 10$ & 9 & 2 \\
\hline \multicolumn{4}{|l|}{ Left } \\
\hline \multirow[t]{2}{*}{ Upper lobe } & $\mathrm{S} 1+2+\mathrm{S} 3$ & 6 & 14 \\
\hline & $\$ 4+5$ & 4 & 8 \\
\hline \multirow[t]{3}{*}{ Lower lobe } & $58+\$ 9$ & 4 & 4 \\
\hline & $S 9+\$ 10$ & 5 & 2 \\
\hline & $\mathrm{S} 8+\mathrm{S} 9+\mathrm{S} 10$ & 7 & 1 \\
\hline Total & & & 41 \\
\hline
\end{tabular}

SS: subsegment.
Table 4 Patients' characteristics of combined subsegmentectomy, single segmentectomy, and multiple segmentectomy

\begin{tabular}{lccc}
\hline & CSS & Single S & Multiple S \\
\hline Mean age (y.o.) & $61 \pm 9$ & $67 \pm 11$ & $71 \pm 8$ \\
& & & \\
Sex & & & \\
$\quad$ Male & 8 & 25 & 16 \\
$\quad$ Female & 9 & 31 & 25 \\
& & & \\
Pulmonary function & & & \\
VC (L) & $3.2 \pm 0.7$ & $3.0 \pm 0.7$ & $3.0 \pm 0.9$ \\
\%VC & $111 \pm 12$ & $100 \pm 13$ & $112 \pm 18$ \\
FEV ${ }_{1}(\mathrm{~L})$ & $2.4 \pm 0.6$ & $2.1 \pm 0.5$ & $2.0 \pm 0.6$ \\
FVC/FEV & & & \\
& $75 \pm 7$ & $75 \pm 7$ & $71 \pm 12$ \\
Mean tumor size (cm) & $1.4 \pm 0.5$ & $1.7 \pm 0.8$ & $2.0 \pm 0.8^{\dagger}$
\end{tabular}

Location of tumor

Right upper lobe

Right lower lobe

Left upper lobe

Left lower lobe

Mean number of resected

subsegments

$\begin{array}{ccc}9 & 17 & 2^{+\dagger} \\ 3 & 15 & 10 \\ 4 & 17 & 22 \\ 1 & 7 & 7\end{array}$

Mean number of preserved subsegments

\begin{tabular}{llll}
\hline Total & 17 & 56 & 41
\end{tabular}

CSS: combined subsegmentectomy, Single S: single segmentectomy, Multiple S: multiple segmentectomy, VC: vital capacity, FVC: functional vital capacity, $\mathrm{FEV}_{1}$ : forced expiratory volume in 1 second.

$t: p=0.002$ between the CSS and the multiple segmentectomy, $+\uparrow: p<0.001$ between the CSS and the multiple segmentectomy.

upper lobe to preserve fewer subsegments than that for other lobes; and (2) Right upper lobe was the resected site more frequent in the CSS than in the multiple segmentectomy ( 53 vs. $5 \%, \mathrm{p}<0.001$ ), causing the discrepancy of numbers of resected and preserved subsegments between the two groups.

Figure 3 shows the mean percentage of preserved $\mathrm{FEV}_{1}$ of whole lung after surgery in the three groups. In the CSS group, the mean values of $\mathrm{FEV}_{1}$ of the whole lung before and after surgery were $2.4 \pm 0.6$ and $2.2 \pm 0.5$ 1 , respectively, of which the mean percentage of $\mathrm{FEV}_{1}$ preserved, was $91 \pm 7 \%$. In the single segmentectomy group, the mean values of $\mathrm{FEV}_{1}$ of the whole lung before and after surgery were $2.2 \pm 0.6$ and $2.0 \pm 0.5 \mathrm{l}$, respectively, of which the mean percentage of $\mathrm{FEV}_{1}$ preserved was $92 \pm 8 \%$. In the multiple segmentectomy group, the mean values of $\mathrm{FEV}_{1}$ of the whole lung before and after surgery were $2.0 \pm 0.6$ and $1.8 \pm 0.6 \mathrm{l}$, respectively, of which the mean percentage of $\mathrm{FEV}_{1}$ 


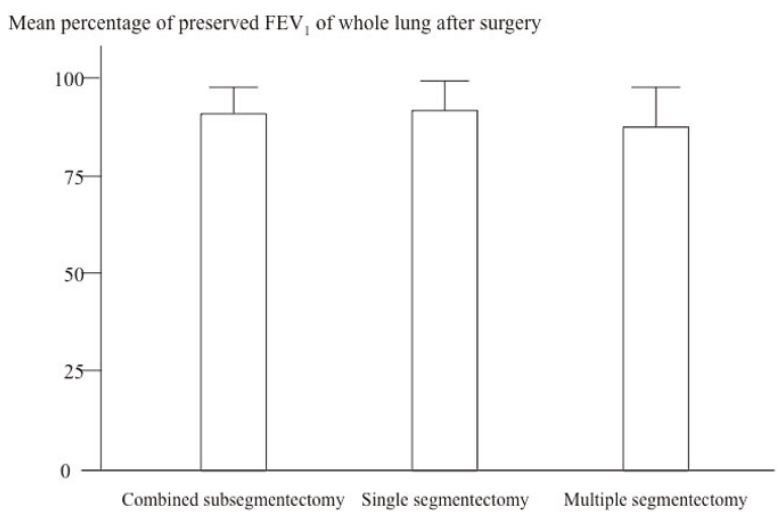

Figure 3 Forced expiratory volume in 1 second examined by pulmonary function tests before and after surgery.

preserved was $88 \pm 10 \%$. No significant difference of the mean percentage of $\mathrm{FEV}_{1}$ preserved was found among these three groups.

Figure 4 shows the $\mathrm{FEV}_{1}$ of each preserved lobe after surgery in the three groups. In the CSS group, the mean values of $\mathrm{FEV}_{1}$ of each lobe before and after surgery were $0.6 \pm 0.2$ and $0.3 \pm 0.2 \mathrm{l}$, respectively. In the single segmentectomy group, the mean values of $\mathrm{FEV}_{1}$ of each lobe before and after surgery were $0.5 \pm 0.2$ and $0.3 \pm$ $0.1 \mathrm{l}$, respectively. In the multiple segmentectomy group, the mean values of $\mathrm{FEV}_{1}$ of each lobe before and after surgery were $0.5 \pm 0.2$ and $0.2 \pm 0.21$, respectively. While there was no significant difference of the postoperative $\mathrm{FEV}_{1}$ of each lobe between the CSS and single segmentectomy, the value of the CSS was higher than that of the multiple segmentectomy with marginal significance $(\mathrm{p}=0.07)$.

Figure 5 shows the $\mathrm{FEV}_{1}$ of each preserved lobe per subsegment after surgery, which were $0.05 \pm 0.03,0.04$ $\pm 0.03,0.04 \pm 0.03 \mathrm{l}$ in the CSS, single segmentectomy, and multiple segmentectomy, respectively. The value

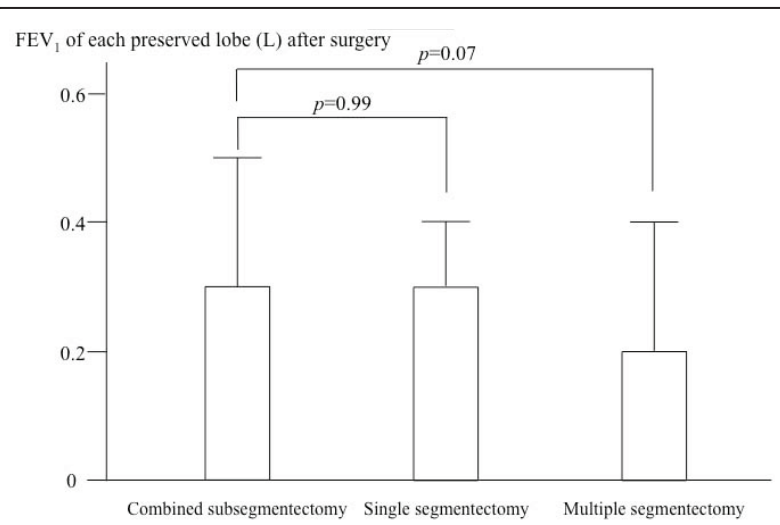

Figure 4 Forced expiratory volume in 1 second of each lobe after surgery.

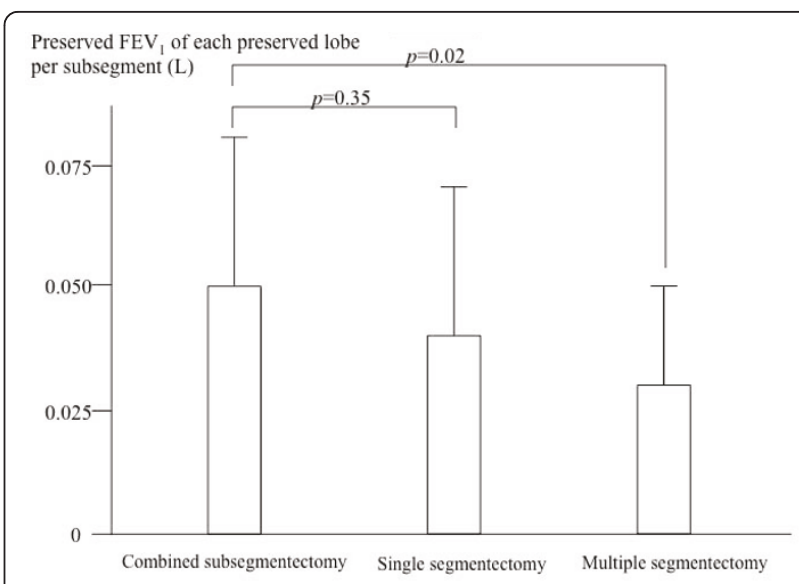

Figure 5 Preserved forced expiratory volume in 1 second of each lobe per subsegment after surgery

was significantly higher in the CSS than in the multiple segmentectomy $(\mathrm{p}=0.02)$.

All of the 198 patients who underwent CSS, single segmentectomy, or multiple segmentectomy were discharged from the hospital without major complications. All of the tumors were pathologically NO stage. With the mean follow-up period after surgery was $31 \pm 10$ months (range: 12-60 month), 5 of the 166 patients (2\%) who underwent single or multiple segmentectomy suffered postoperative recurrence, but there was no recurrence at the surgical margin. All 32 patients who underwent CSS are alive without recurrence.

\section{Discussion}

Results of this study elucidated the following points: (1) The CSS could save $2.2 \pm 1.2$ subsegments compared with the resection of entire segments involved by tumors; and (2) Both the preserved $\mathrm{FEV}_{1}$ of each lobe and that value per subsegment were higher in the CSS than in the multiple segmentectomy, whereas there was no significant difference of preserved $\%$ of $\mathrm{FEV}_{1}$ of whole lung between the two groups.

The reason for no significant difference in pulmonary function between the CSS and multiple segmentectomy could be caused by the difference of frequency of right upper lobe between the two. The CSS was conducted for right upper lobe more frequently than the multiple segmentectomy, because the right upper lobe has fewer subsegments than the other lobes. To preserve sufficient lung tissue for tumors involving multiple segments in the right upper lobe, we conducted the CSS frequently, for example the resection of S2b and S3a rather than the resection of both the S2 and S3. Contrary to the right upper lobe, other lobes can preserve sufficient lung tissue even after multiple segmentectomy, because they have more subsegments than the right upper lobe. 
Therefore, our data show that the CSS could preserve the pulmonary function of each lobe by avoiding the multiple segmentectomy, especially for tumors in the right upper lobe.

The mean values of postoperative $\mathrm{FEV}_{1}$ after CSS, single segmentectomy, and multiple segmentectomy were approximately $90 \%$ of the preoperative values, which were comparable to values in the previous reports of general segmentectomy $[7,12,13]$ and were higher than that after lobectomy [12]. We previously reported that the postoperative $\mathrm{FEV}_{1}$ of each lobe after the resection of 1,2 , and 3 segments was decreased to $50 \%, 35 \%$, and $17 \%$, respectively [7]. The use of CSS can obviate the resection of multiple segments that a tumor involves. Therefore, to preserve a pulmonary function after segmentectomy in patients with small peripheral c-T1N0M0 NSCLC involving multiple segments, CSS would be preferable to the resection of multiple segments with tumor involvement, especially for small tumors located in the right upper lobe.

This study revealed that the mean tumor size in the CSS was significantly smaller than that in multiple segmentectomy. The mean tumor size in the CSS group was $1.4 \pm 0.5 \mathrm{~cm}$, ranging from 0.8 to $2.4 \mathrm{~cm}$. To take the surgical margin of at least $2 \mathrm{~cm}$ from the tumor by the CSS, tumors larger than $2 \mathrm{~cm}$ involving multiple segments would be out of the indication for CSS.

The disadvantage of the CSS might be that the lymph node dissection at hilum of resected subsegments would be less sufficient than the conventional segmentectomy. Therefore, we recommend it for likely pathological NO tumors, such as bronchioloalveolar carcinoma, carcinoid, and metastatic pulmonary tumors.

The preserved pulmonary functions after CSS, single segmentectomy, and multiple segmentectomy are shown herein. Our data indicate that the CSS is useful for preservation of pulmonary function of each lobe by avoiding the multiple segmentectomy especially in patients with small sized tumors with likely pathological N0 involving multiple segments of the right upper lobe.

\section{Abbreviations}

NSCLCs: non-small cell lung cancers; CSS: combined subsegmentectomy; $\mathrm{FEV}_{1}$ : forced expiratory volume in 1 second; SPECT/CT: lung-perfusion singlephoton-emission computed tomography and computed tomography; RI: radioisotope.

\section{Author details}

'Departments of Thoracic Surgery, Faculty of Life Sciences, Kumamoto University, 1-1-1 Honjo, Kumamoto 860-8556, Japan. ²Division of General Thoracic Surgery, Department of Surgery, School of Medicine, Keio University, Tokyo, Japan.

\section{Authors' contributions}

This report reflects the opinion of the authors and does not represent the official position of any institution or sponsor. The contributions of each of the authors were as follows: $\mathrm{KY}$ was responsible for reviewing previous

research, journal handsearching, drafting report. HN was responsible for quality checking and data processing. HN was responsible for project coordination. All authors have read and approved the final manuscript.

\section{Competing interests}

The authors declare that they have no competing interests.

Received: 12 December 2010 Accepted: 20 February 2011

Published: 20 February 2011

\section{References}

1. Okada M, Koike T, Higashiyama M, Yamato Y, Kodama K, Tsubota N: Radical sublobar resection for small-sized non-small cell lung cancer: a multicenter study. J Thorac Cardiovasc Surg 2006, 132:769-75.

2. Yoshikawa K, Tsubota N, Kodama K, Ayabe H, Taki T, Mori T: Prospective study of extended segmentectomy for small lung tumors: The final report. Ann Thorac Surg 2002, 73:1055-9.

3. Kodama K, Doi O, Higashiyama M, Yokouchi H: Intentional limited resection for selected patients with T1N0M0 non-small cell lung cancer. J Thorac Cardiovasc Surg 1997, 114:347-53.

4. Koike T, Yamato $Y$, Yoshiya K, Shimoyama T, Suzuki R: Intentional limited pulmonary resection for peripheral T1N0M0 small-sized lung cancer. $J$ Thorac Cardiovasc Surg 2003, 125:924-8.

5. Yoshimoto K, Nomori H, Mori T, Kobayashi H, Ohba Y, Shibata H, Shiraishi S, Kobayashi T: Prediction of pulmonary function after lung lobectomy by subsegments counting, computed tomography, single photon emission computed tomography and computed tomography: a comparative study. Eur J Cardiothorac Surg 2009, 35:408-13.

6. Ohno Y, Koyama H, Takenaka D, Nogami M, Kotani Y, Nishimura Y, Yoshimura M, Yoshikawa T, Sugimura K: Coregistered ventilation and perfusion SPECT using krypton-81 $\mathrm{m}$ and Tc-99m-labeled macroaggregated albumin with multislice $C T$ utility for prediction of postoperative lung function in non-small cell lung cancer patients. Acad Radiol 2007, 14:830-838.

7. Yoshimoto K, Nomori H, Mori T, Kobayashi H, Ohba Y, Shibata H, Tashiro K, Shiraishi S, Kobayashi T: Quantification the Impact of Segmentectomy on Pulmonary Function by Perfusion SPECT/CT. J Thorac Cardiovasc Surg 2009, 137:1200-5.

8. Nomori H, Ikeda K, Mori T, Kobayashi H, Iwatani K, Kawanaka K, Shiraishi S, Kobayashi T: Sentinel node navigation segmentectomy for c-T1N0M0 non-small cell lung cancer. J Thorac Cardiovasc Surg 2007, 133:780-785

9. Nomori H, Ohba Y, Shibata H, Shiraishi K, Mori T, Shiraishi S: Required area of lymph node sampling during segmentectomy for clinical stage IA non-small cell lung cancer. J Thorac Cardiovasc Surg 2010, 139:38-42.

10. Tsubota N: An improved method for distinguishing the intersegmental plane of the lung. Surg Today 2000, 30:963-4

11. American Thoracic Society: Standardization of spirometry - 1987 update. Am Rev Respir Dis 1987, 136:1285-98.

12. Harada H, Okada M, Sakamoto T, Matsuoka H, Tsubota N: Functional advantage after radical segmentectomy versus lobectomy for lung cancer. Ann Thorac Surg 2005, 80:2041-5.

13. Sienel W, Dango S, Kirschbaum A, Cucuruz B, Horth W, Stremmel C, Passlick B: Sublobar resections in stage IA non-small cell lung cancer: segmentectomies result in significantly better cancer-related survival than wedge resections. Eur J Cardiothorac Surg 2008, 33:728-34.

doi:10.1186/1749-8090-6-17

Cite this article as: Yoshimoto et al: Combined subsegmentectomy: postoperative pulmonary function compared to multiple segmental resection. Journal of Cardiothoracic Surgery 2011 6:17. 